\title{
Spatial Motion Constraints for Robot Assisted Suturing Using Virtual Fixtures
}

\author{
Ankur Kapoor, Ming Li, and Russell H. Taylor \\ Dept. of Computer Science, Johns Hopkins University
}

\begin{abstract}
We address the problem of the stitching task in endoscopic surgery using a circular needle under robotic assistance. Our main focus is to present an algorithm for suturing using guidance virtual fixtures (VF) that assist the surgeon to move towards a desired goal. A weighted multi-objective, constraint optimization framework is used to compute the joint motions required for the tasks. We show that with the help of $\mathrm{VF}$, suturing can be performed at awkward angles without multiple trials, thus avoiding damage to tissue. In this preliminary study we show the feasibility of our approach and demonstrate the promise of cooperative assistance in complex tasks such as suturing.
\end{abstract}

\section{Introduction}

The benefits of Minimally Invasive Surgery (MIS) over conventional open surgery are well known, however, the surgeon faces the challenge of limited and constrained motion as well as loss of direct visualization. These factors make the simple act of suturing probably the most difficult and time consuming of all MIS tasks. For these reasons, robotic assisted MIS has gained increasing popularity during the last decade. As shown in [1] by Ruurda et al. there is a significant improvement in time needed per stitch with robotic assistance even for experienced surgeons. Hubens 2] reaches a similar conclusion for standardized surgical tasks performed by inexperienced surgeons. To further augment the surgeon's ability to manipulate the surgical instruments using robotic system in a confined environment, various techniques have been proposed in literature. In [3] the surgeon's view is enhanced by $3 \mathrm{D}$ vision. Kitagawa et al. [4] provide force feedback through sensory substitution to achieve suture results that are closer to ideal conditions. It is important to note that in the various surgical systems described above the surgical procedures are still performed by surgeon; the robotic device merely follows the human commands. Estimation of distances and angles with a "key hole" view provided by endoscopic cameras becomes difficult and time consuming for surgeons. Consequently, the suturing motion is often realized by multiple trials that extend the operating time as pointed out by Ruurda et al. [1]. This indicates that a robotic assistant system that uses surgeon's intelligence for high level cognition tasks and at the same time fills the gap in sensory perception by providing motion guidance will be useful.

Different techniques 5678 , have been proposed to provide interaction modes in which the surgeon shares the control of the robot with the computer process. 
The goal of the computer process is to provide anisotropic motion behavior to the surgeon's motion command besides filtering out tremor and disturbance to enhance precision and stability. Li and Taylor 9] extended Funda's work [10 to generate virtual fixtures (VF) to assist the surgeon to manipulate surgical tools in a complex work space environment, in which anatomical constraints are automatically generated from 3D medical images.

The suturing task was observed and analyzed as performed in training videos. This task involves the following steps 1) (Select) Determine a suitable entry and exit point for the suture needle leaving sufficient room from the edge to be approximated. 2) (Align) Grasp the needle, move and orient it such that the tip is aligned with the entry point. 3) (Bite) Entry and exit "bites" are made such that the needle passes from one tissue to be approximated to the other. 4) (Loop) Create a suture loop to tie a knot. 5) (Knot) Secure the knot under proper tension. Previous works have focuses on steps 4 and 5 and used a shuttle device to address the issue of manipulation of curved needle. Kang and Wen [1] and Nagy et al. 12 have focused on the knot tying aspects of suturing. Both of them use tele-manipulation with haptic feedback to perform these tasks. Nageotte et al. 13. have presented a kinematic analysis of the entrance and exit bites involved in stitching task. In this work we describe our recent approach to VF that can combine guidance with forbidden regions relative to features on the target and its application to the task of stitching.

\section{Methods}

In this work we address the align and bite steps of the suturing process described above, where the primary challenges are manipulation of curved needle under non-ideal haptic using a robot with complex kinematics. In the align step, the goal is to move the robot to align the position and orientation of the suture needle such that it pierces the tissue correctly, at the same time minimizing extraneous motion of the needle and robot. The goal of the bite step is to move the needle tip from entry point to the exit point with minimum damage to the tissue through which the needle passes.

\subsection{Constrained Control Algorithm Overview}

For this work, we assume that the robot is holding the needle and the needle tip and target have been registered in the robot coordinate system. The outline of the algorithm is as follows: 1) Obtain the incremental motion desired by the user through force sensor, joystick or master 2) Formulate a set of linear constraints based on current robot state and specified task 3) Use the robot and task instantaneous kinematics to generate a quadratic program with linear constraints. The general form of the program is

$$
\begin{gathered}
\arg \min _{\Delta q}\left\|W\left(\Delta \boldsymbol{x}-\Delta \boldsymbol{x}^{d}\right)\right\|, \\
\text { s.t. } \quad H \Delta \boldsymbol{x} \geq \boldsymbol{h}, \quad \Delta \boldsymbol{x}=J \Delta \boldsymbol{q}
\end{gathered}
$$

where $\Delta \boldsymbol{q}$ is the desired incremental motions of the joint variables, $\Delta \boldsymbol{x}^{d}, \Delta \boldsymbol{x}$ are the desired and the computed incremental motions of the task variables 
in Cartesian space, respectively. $J$ is the Jacobian matrix relating task space to joint space. $W$ is a diagonal matrix for weights selected so that the errors of critical motion elements are close to zero, while errors in other non-critical motions simply stay as low as possible within tolerances allowed by the constraint set. 4) Solve the quadratic program for the incremental joint motion, which is used to move the robot. We would like to note that the constraints of step 3 might not be linear such as the distance function. In such cases we use a linear approximation, which allows us to utilize the structure of least squares problem with linear constraints, and solve the quadratic program in time frames suitable for robot control. We have used the Lawson and Hanson's algorithm as presented in $[14$.

\subsection{Modeling of Task}

We assume that the entry and exit points are known in the robot coordinate frame. These could be specified by surgeon using an optical marker tool to indicate points in space or by using a computer vision system to determine suitable points on the surface based on distance from the edge to be approximated and transferred to the robot coordinate frame (Reader is refered to [15] for a general purpose toolkit for computer vision).For the purpose of this work we use a point based registration method using Optotrak. We shall denote $\boldsymbol{p}_{i}$ as the position of the origin of frame $\{i\}$ with respect to world coordinates, where $\{i\}$ is any one of the frames shown in Figure 1.

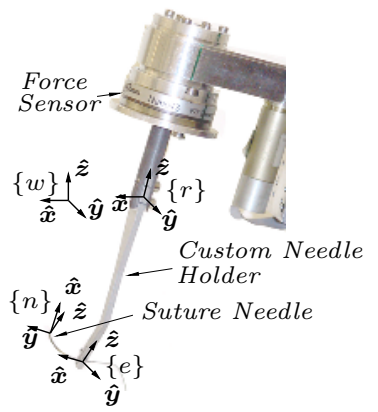

Fig. 1. Custom needle holder, needle and assigned frames
Align Step. We now present a strategy that could be used for the align step with the following substeps. (Substep 1) First the needle tip is allowed to move in a straight line such that the needle tip coincides with the desired entry point; at the same time its orientation is allowed to change only about an axis such that this motion will result in the tangent at needle tip being coincident with the normal to the surface at the entry point. (Substep 2) In the next substep the orientation of the normal to the needle plane is allowed to change, such that the needle plane coincides with line joining entry and exit points. Assistance is provided by not allowing any motion of needle tip or the tangent at the needle tip. (Substep 3) Once the desired orientations are reached we allow the surgeon to penetrate the tissue by a small distance, (Substep 4) followed by motion constraints that would let the surgeon bring the tangent at needle tip to coincide with the desired entry direction without changing the plane normal and tip position. The align step is completed once the desired orientation is reached, which is computed using the entry and exit points specified by the surgeon and the needle radius. In all these substeps only those motions that bring the needle closer to the desired position and orientation are allowed. 
The above sequence of substeps ensures that the needle tip (cutting point) is normal to the tissue surface at the time of piercing the tissue. This is particularly favorable for hard tissue such as muscle as it makes the optimal use of the cutting point of needle. In case of soft tissue, the surgical needles used do not have a sharp cutting point. Thus in order to reduce the number of substeps and hence the time, an alternate strategy in which the substeps are to align needle tip directly with desired direction instead of surface normal and skiping the last two substeps could be used. For the remainder of this paper, we shall focus on the former approach. For our algorithm, the later approach can be considered as a special case of former.

Moreover, for the current work we ignore the deformation in the tissue. For the future we want to extend this approach by regarding the deformation and updating the targets accordingly.

Bite Step. (Substep 5) Once the entry and exit points are determined, and the radius of needle is known, it easy to see that the trajectory of the needle tip that would cause minimum damage to the tissue lie on a circle with entry and exit points as points on a chord and with radius equal to needle radius. To ensure sufficient depth of penetration in the tissue we ensure that the needle plane is parallel to the line joining entry and exit points and the surface normal at entry point. In this step our constraint motion algorithm permits only those motions that satisfy these constraints.

\subsection{Algorithm Implementation}

In our approach the required VF constraints for each substep are analyzed and broken into a combination of one or more of basic constraints.

We make use of the common structure be-

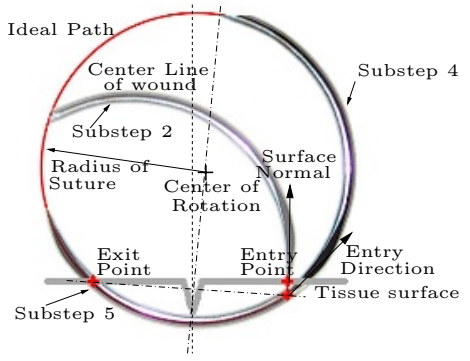

Fig. 2. Ideal path of needle tip for minimal tissue tear is a circle with the same radius as the suture needle and centered at needle center tween the different substeps and construct generalized constraints that take desired target into consideration. Furthermore we utilize the sequential nature of the task to switch between different substeps. The switch could be triggered when the error between the current value and target decreases below a threshold. We now describe a method to compute $H$ and $\boldsymbol{h}$ in the inequality subject function of (1) corresponding to one of basic constraints, then we show how we can combine then together to form the desired VF behavior. We use MOVE (and ROTATE) for the constraint of moving along a desired direction (or rotate about a desired direction). We use the name STAY (and MAINTAIN) for a VF designed to maintain a desired position (or orientation). We denote signed error for target by $\boldsymbol{\delta}$, its translational part by $\boldsymbol{\delta}_{p}$ and the rotation component by a Rodriguez vector $\boldsymbol{\delta}_{r}$. Since the angles are small, we can approximate the Rodriguez vector by Euler angles. 
STAY Constraint. We are given a desired target point $\boldsymbol{p}_{i}^{d}$ in some frame $\{i\}$. This gives signed error as $\boldsymbol{\delta}=\left[\boldsymbol{p}_{i}-\boldsymbol{p}_{i}^{d}, 0\right]^{t}$. We require that after incremental motion due to input, the position be as close as possible to the target point. This requires that $\left\|\boldsymbol{\delta}_{p}+\Delta \boldsymbol{p}_{i}\right\| \leq \epsilon_{1}$ where $\epsilon_{1}$ is a small positive number that defines the size of the range that can be considered as the target. To gain computational efficiency we would like to solve a quadratic program with linear constraints. Thus, we linearize the above constraint by considering projections of $\boldsymbol{\delta}_{p}+\Delta \boldsymbol{p}_{i}$ on a finite set of lines through target point. These can be written as

$$
\begin{gathered}
{\left[c_{\alpha i} c_{\beta j}, c_{\alpha i} s_{\beta j}, s_{\alpha i}, 0,0,0\right]^{t}(\boldsymbol{\delta}+\Delta \boldsymbol{x}) \leq \epsilon_{1} ; \quad i=1, \cdots, n ; j=1, \cdots, m .} \\
\text { where } \quad c_{\alpha i}=\cos \frac{2 \pi i}{n} ; c_{\beta j}=\cos \frac{2 \pi j}{m} ; s_{\alpha i}=\sin \frac{2 \pi i}{n} ; s_{\beta j}=\sin \frac{2 \pi j}{m}
\end{gathered}
$$

Then we set $H \in \Re^{6 \times m n}$ and $\boldsymbol{h} \in \Re^{m n}$ as

$$
H=\left[\begin{array}{ccc}
-c_{\alpha 1} c_{\beta 1}, & -c_{\alpha 1} s_{\beta 1}, & -s_{\alpha 1}, 0,0,0 \\
\cdots & \cdots \\
-c_{\alpha 1} c_{\beta m}, & -c_{\alpha 1} s_{\beta m}, & -s_{\alpha 1}, 0,0,0 \\
\cdots & \cdots \\
-c_{\alpha n} c_{\beta 1}, & -c_{\alpha n} s_{\beta 1}, & -s_{\alpha n}, 0,0,0 \\
-c_{\alpha n} c_{\beta m}, & -c_{\alpha n} s_{\beta m}, & -s_{\alpha n}, 0,0,0
\end{array}\right], \quad \boldsymbol{h}=\left[\begin{array}{c}
-\epsilon_{1} \\
\vdots \\
-\epsilon_{1}
\end{array}\right]-H \boldsymbol{\delta}
$$

MOVE Constraint. We are given a desired line $L(s)=\boldsymbol{L}_{0}+\hat{\boldsymbol{l}} \cdot s$ in some frame $\{i\}$. We require that the position after incremental motion due to input be as close to the line as possible. If $\boldsymbol{p}_{i}^{c}$ is the closest point on the line to the current position, then the signed error is $\boldsymbol{\delta}=\left[\boldsymbol{p}_{i}-\boldsymbol{p}_{i}^{c}, 0\right]^{t}$. We define $\boldsymbol{u}_{\boldsymbol{i}}$ as the projection of $\boldsymbol{p}_{i}-\boldsymbol{p}_{i}^{c}$ on a plane $\Pi$ that is perpendicular to the line. Our requirement is that $\left\|\boldsymbol{u}_{i}\right\|$ be close to zero. If $R$ denotes a

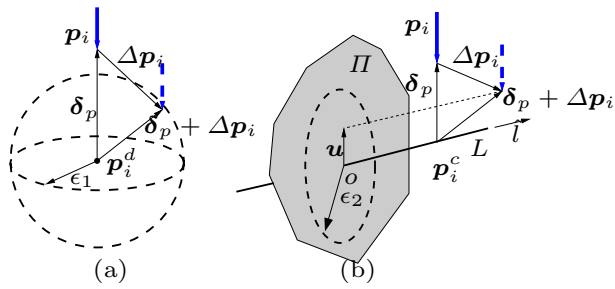

Fig. 3. Geometric relation for (a) STAY and (b) MOVE rotation matrix that would transform a vector in plane $\Pi$ to world coordinates, then any unit vector in plane $\Pi$ with $O$ (Figure 3) as origin can be written in world coordinate frame as $R[\cos \gamma, \sin \gamma, 0]^{t}$.

Like before we approximate by considering only a finite set of vectors, and our constraint can be written as

$$
\left[\left[R\left[c_{\gamma i}, s_{\gamma i}, 0\right]^{t}\right]^{t}, 0,0,0\right]^{t}(\boldsymbol{\delta}+\Delta \boldsymbol{x}) \leq \epsilon_{2} ; \quad c_{\gamma i}=\cos \frac{2 \pi i}{k} ; s_{\gamma i}=\sin \frac{2 \pi i}{k} ; i=1, \cdots, k
$$

We can set $H \in \Re^{6 \times k}$ and $\boldsymbol{h} \in \Re^{k}$ as,

$$
H=\left[\begin{array}{c}
-\left[R\left[c_{\gamma 1}, s_{\gamma 1}, 0\right]^{t}\right]^{t}, 0,0,0 \\
-\left[R\left[c_{\gamma k}, s_{\gamma k}, 0\right]^{t}\right]^{t}, 0,0,0
\end{array}\right], \quad \boldsymbol{h}=\left[\begin{array}{c}
-\epsilon_{2} \\
\cdots \\
-\epsilon_{2}
\end{array}\right]-H \boldsymbol{\delta}
$$

The construction of $H$ and $\boldsymbol{h}$ for MAINTAIN and ROTATE follow the same lines, and uses the later three columns of matrix $H$ corresponding to three rotational 
components in $\Delta \boldsymbol{x}$. We refer the reader to [16] for further details on contruction of matrix $H$ for different constraints. A combined constraint can be applied to a same frame or to different frames. For the case of different frames we consider Jacobian corresponding to each frame and the inequality subject function is of the form (6a) where $J_{i}(q)$ is the Jacobian matrix that maps Cartesian velocities of frame $\{i\}$ to joint space. For the case of same frame the inequality subject function of (11) is of the form (6b) where subscript $p$ and $r$ denotes the translational and rotational constraints respectively.

$$
\left[\begin{array}{cc}
H_{1} & 0 \\
\ddots & H_{k}
\end{array}\right]\left[\begin{array}{c}
J_{1}(q) \\
\vdots \\
J_{k}(q)
\end{array}\right] \Delta \boldsymbol{q} \geq\left[\begin{array}{c}
\boldsymbol{h}_{1} \\
\vdots \\
\boldsymbol{h}_{k}
\end{array}\right] \quad(6 \mathrm{a}) \quad\left[\begin{array}{c}
H_{p} \\
H_{r}
\end{array}\right] J(q) \Delta \boldsymbol{q} \geq\left[\begin{array}{c}
\boldsymbol{h}_{p} \\
\boldsymbol{h}_{r}
\end{array}\right]
$$

\section{Materials}

As a preliminary test bed (Figure 4) we have used a Johns Hopkins University Steady-Hand robot [17, which is equipped with a custom needle holder and a 6-DoF force-torque sensor (ATI Nano43 F/T transducer) mounted on the tool handle. For these experiments we have selected a $3 / 8$ circle $30 \mathrm{~mm}$ cutting needle from Ethicon (needle diameter $1 \mathrm{~mm}$ ). The Optotrak (Northern Digital Inc, Waterloo, CA) infrared optical position tracking system was used for robot calibration.Our control algorithm is independent of manipulator type and input form a joystick or a master robot can easily replace the force input to the controller. The phantom to mimic the tissue is contructed by dissolving $4 \%$ by weight of agar in distilled water, maintaing this solution at $90 \operatorname{deg} C$ for one hour and solidifying the solution by rapidly cooling it to $-20 \operatorname{deg} C$.

\section{Experiments}

We performed three sets of experiments; The first set was computer simulation to check the feasiblity of motion given the constraints and robot kinematics. The second set was experiments with the robot. We recoreded the encoder reading of the robot joints and used direct kinematics of the robot to verify our algorithm by measuring the errors between the ideal target path and that followed by the robot. Figure 5 show the errors between actual and ideal robot motion as
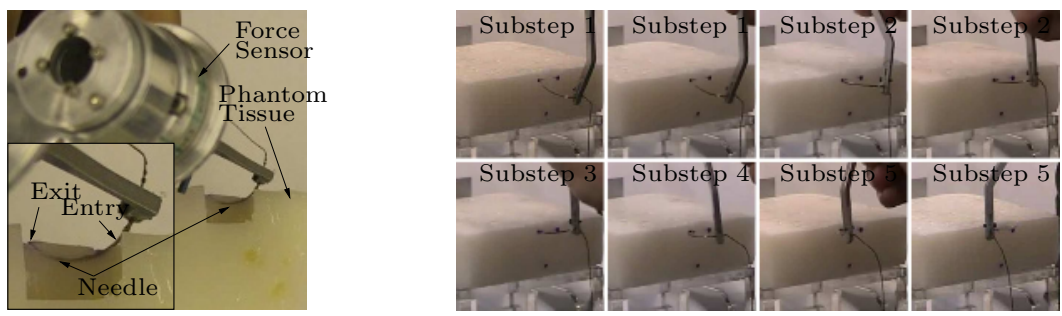

Fig. 4. (left) Experimental Setup, Insert: Path of needle (right) Phantom being sutured 


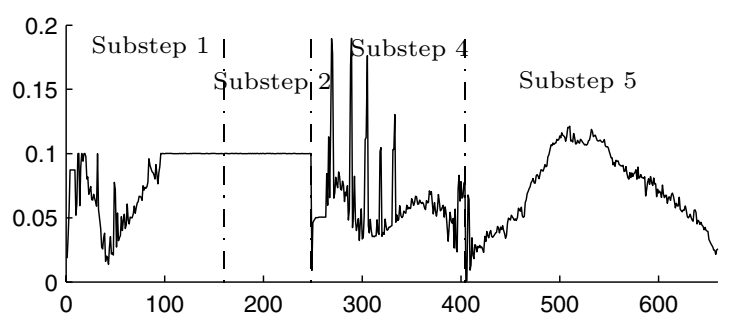

Fig. 5. The magnitude of error in needle tip with respect to ideal path as measured by robot encoders
Table 1. The error in ideal and actual points as measured by OptoTrak

\begin{tabular}{|c|c|c|}
\hline & Entry & Exit \\
\hline \hline Robot & 0.6375 & 0.7742 \\
Manual & - & 2.1 \\
\hline
\end{tabular}

measured with robot encoders and kinematics for different substeps. The values $\left(\epsilon_{i}\right.$ in equations 3 and 5) for positional and angular tolerance were selected as $0.5 \mathrm{~mm}$ and $0.25 \mathrm{deg}$. The diameter of the needle was $1 \mathrm{~mm}$. The last set of experiments were a demonstration of our algorithm using a phantom tissue. Since the phantom is opaque, the measurements available are the entry and exit points of the needle. Table 1 presents the differences between the user specified targets and the actual ones as measured by an optical tool. As expected the errors measured by Optotrak are higher than measured by encoders alone, because this represents the overall accuracy of the system, which also includes errors arising from calibration of the needle and accuracy of Optotrak $(0.1 \mathrm{~mm})$. The residual calibration errors are appear as errors in the entry point errors in Table 1 above. Average errors for free hand suturing as performed by four users ( 5 trials each), using the same needle holder and without robot assistance, are presented in Table 1. We believe that robot assistance can improve accuracy especially in constrained environment such as that of endoscopic surgery. Moreover, robot assisted motions did not require multiple trials and large undesirable movements of tissue, which is often the case in free hand suturing. Figure 4 shows the progression of different substeps for one of the trials. The insert in Figure 4 shows the phantom with a portion cut out so that the actual path taken by the needle is visible, the entry and exit points are $13.5 \mathrm{~mm}$ apart. As seen in the Figure 4 we have selected an angle that places limits on performing the suture manually, to emphasize the ability of our algorithm to assist in non-favorable orientations.

\section{Conclusion}

Endoscopic surgery presents a constrained working environment for surgeons, and the surgeons must deal with the realities of long instruments and awkward angles. In this paper we have implemented the constrained control for performing "align" and "bite" steps, given a surface, entry and exit points. Using guidance virtual fixtures we provide assistance to the surgeon allowing only those motions of the needle that are feasible and move the needle towards the desired goal. This helps realize the stitching motion without multiple trials and large undesirable movements of tissues involved. This formulation can be extended to include additional constraints such as collision avoidance and anatomy-based constraints 
9. We plan to extend these results by integrating vision to take into account deformation and also add force feedback. We are also working on implementing this algorithm for control of a high dexterity snake-like robot [18] geared towards long and slender anatomy such as throat.

\section{Acknowledgements}

We thank Iulian Iordachita for his assistance with the robot and the needle holder. This work was partially funded by the National Science Foundation (NSF) under Engineering Research Center grant \#EEC9731478, NSF grant \#IIS9801684, and by the Johns Hopkins University internal funds.

\section{References}

1. Ruurda, J.P., et al: Manual robot assisted endoscopic suturing: Time-action analysis in an experimental model. Surg. End. 18 (2004) 1249-1252

2. Hubens, G., et al: A performance study comparing manual and robotically assisted laparoscopic surgery using the da vinci system. Surg. End. 17 (2003) 1595-1599

3. Guthart, G., Salisbury, K.: The intuitive TM telesurgery system: Overview and application. In: ICRA. (2000) 618-621

4. Kitagawa, M., et al: Effect of sensory substitution on suture-manipulation forces for robotic surgical systems. J Thorac Cardiovasc Surg 129 (2005) 151-158

5. Kazanzides, P., et al: Force sensing and control of a surgical robot. In: Trans. Robot. Automat. (1992)

6. Rosenberg, L.B.: Virtual fixtures: Perceptual tools for telerobotic manipulation. In: IEEE Virtual Reality Annual International Symposium. (1993) 76-82

7. Davies, B.L., et al: Active compliance in robotic surgerythe use of force control as a dynamic constraint. Proc Inst Mech Eng [H]. 211 (1997) 285-292

8. Dewan, M., et al: Vision-based assistance for ophthalmic micro-surgery. In: MICCAI. (2004)

9. Li, M., Taylor, R.H.: Spatial motion constraints in medical robot using virtual fixtures generated by anatomy. In: ICRA. (2004) 1270-1275

10. Funda, J., et al: Constrained cartesian motion control for teleoperated surgical robots. Trans. Robot. Automat. 12 (1996) 453-465

11. Kang, H., Wen, J.T.: Endobot: a robotic assistant in minimally invasive surgeries. In: ICRA. (2001) 2031-2036

12. Nagy, I., et al: Endo[PA]R: An open evaluation system for minimally invasive robotic surgery. In: Mechatronics and Robotics. (2004)

13. Nageotte, F., et al: Computer-aided suturing in laparoscopic surgery. In: CARS. (2004) 781-786

14. Lawson, C.L., Hanson, R.J.: Solving Least Squares Problems. Prentice-Hall, Inc. (1974)

15. Hager, G., Toyama, K.: The xvision system: A general-purpose substrate for portable real-time vision applications. CVIU 69 (1998) 23-37

16. Li, M., Kapoor, A., Taylor, R.: A constrained optimization approach to virtual fixtures. In: ICRA. (2005) Submitted for review Submitted for review.

17. Taylor, R.H., et al: A steady-hand robotic system for microsurgical augmentation. IJRR 18 (1999) 1201-1210

18. Simaan, N., et al: High dexterity snake-like robotic slaves for minimally invasive telesurgery of the upper airway. In: MICCAI. (2004) 